\title{
The discovery and dynamical evolution of an object at the outer edge of Saturn's A ring
}

\author{
Carl D. Murray ${ }^{*, a}$, Nicholas J. Cooper ${ }^{\mathrm{a}}$, Gareth A. Williams ${ }^{\mathrm{a}}$, Nicholas O. Attree ${ }^{\mathrm{a}}$, Jeffrey S. Boyer ${ }^{\mathrm{b}}$ \\ ${ }^{a}$ Queen Mary University of London, Astronomy Unit, Mile End Road, London E1 4NS, UK \\ ${ }^{b}$ Jet Propulsion Laboratory, California Institute of Technology, Pasadena, California, USA
}

\begin{abstract}
We report on the serendipitous discovery of an unresolved, evolving, sub-km-radius object with a semimajor axis $<10 \mathrm{~km}$ inside that of the edge of Saturn's main rings. The object has been detectable in Cassini images since at least May 2012 and its changing orbit shows evidence of a possible disruption in early 2013.
\end{abstract}

Key words: Saturn, rings, Satellites, dynamics, Resonances, orbital

\section{Introduction}

Saturn's rings are a conveniently located dynamical laboratory in which to study how objects embedded in an astrophysical disk form and evolve (Cuzzi et al., 2010). Ring systems may also be the place where planetary satellites form and migrate outwards (Charnoz, Salmon \& Crida (2010), Crida \& Charnoz (2012)). Saturn's A ring contains two moons, Pan and Daphnis (mean radius $14 \mathrm{~km}$ and $4 \mathrm{~km}$ respectively (Thomas et al., 2013)), maintaining the Encke and Keeler gaps, as well as smaller objects (radii $<1 \mathrm{~km}$ ) producing characteristic 'propeller' structures by perturbing adjacent ring particles (Tiscareno et al. (2006), Tiscareno et al. (2008)). The largest of these structures is known to be undergoing orbital evolution (Tiscareno et al., 2010) and its unresolved, associated moonlet may eventually escape from the main rings. Current observations of "propellers' by the Cassini spacecraft are key to understanding the competing roles of ring torques and stochastic processes in determining the migration of embedded masses (Crida et al., 2010).

\footnotetext{
*Corresponding author

Email address: C.D.Murray@qmul.ac.uk (Carl D. Murray)
}

\section{Discovery}

An image of the moon Prometheus together with Saturn's A and F rings taken by the Cassini spacecraft's Imaging Science Subsystem (Porco et al., 1984) (ISS) on 2013 April 15 revealed the presence of a bright, extended feature at the edge of the A ring and seemingly detached from it (Fig. 1). The discovery image was one of a pair taken $33 \mathrm{~s}$ apart with exposure durations of $680 \mathrm{~ms}$ and $150 \mathrm{~ms}$; the feature was apparent in both images thereby ruling out the possibility of it being a cosmic ray artefact. It had a maximum radial width of $\sim 10 \mathrm{~km}$ at its brightest point with a longitudinal extent of $\sim 0.5^{\circ}$ (equivalent to $\sim 1,200 \mathrm{~km}$ at this radial location); it was $\sim 20 \%$ brighter than immediately adjacent ring material. In terms of morphology, relative brightness and extent, no similar feature has been detected in Saturn's main rings.

With the assumption of an elliptical, keplerian orbit consistent with its observed radial location in the discovery image, a list of existing images that could contain the object was produced. This list was augmented as new images were received from the spacecraft. We also examined all images containing the edge of the A ring from our existing 


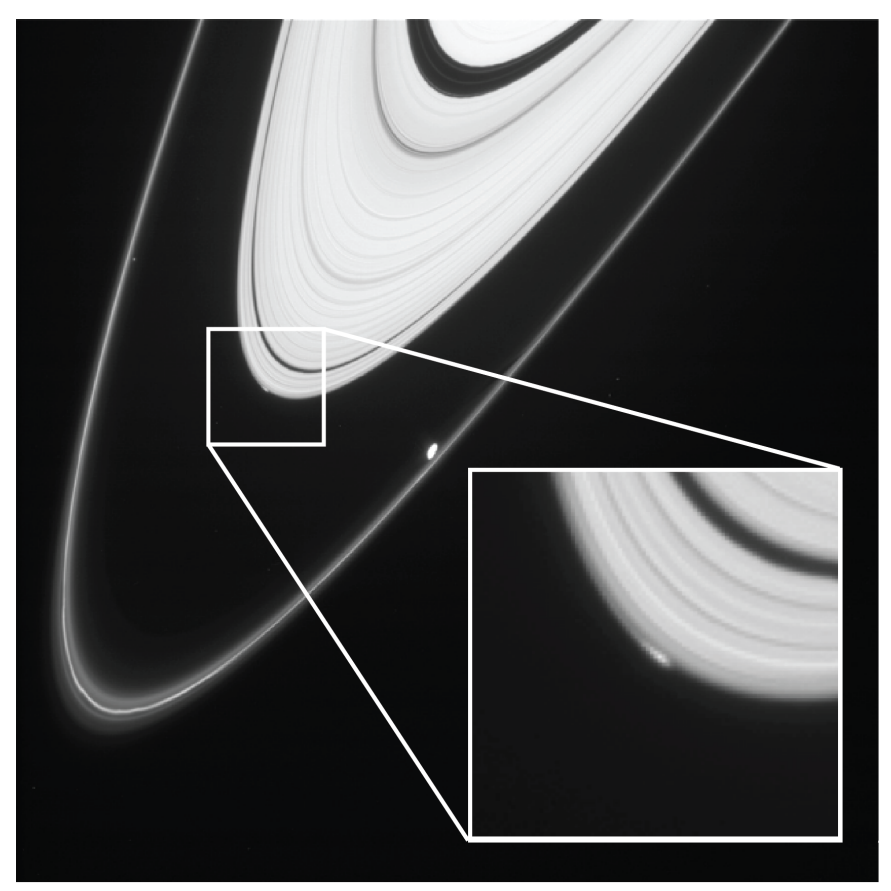

Figure 1: Discovery image taken by Cassini ISS narrow angle camera on 2013 April 15. Image N1744738746 and enlargement of ansa region showing extended object $10 \mathrm{~km}$ beyond the edge of the A ring. The $680 \mathrm{~ms}$ exposure was targeted at Prometheus (center of image) and is one of a series designed to improve the orbits of the small satellites. A radius-longitude reprojected image of the ansa region is shown in Fig. $2 \mathrm{f}$.

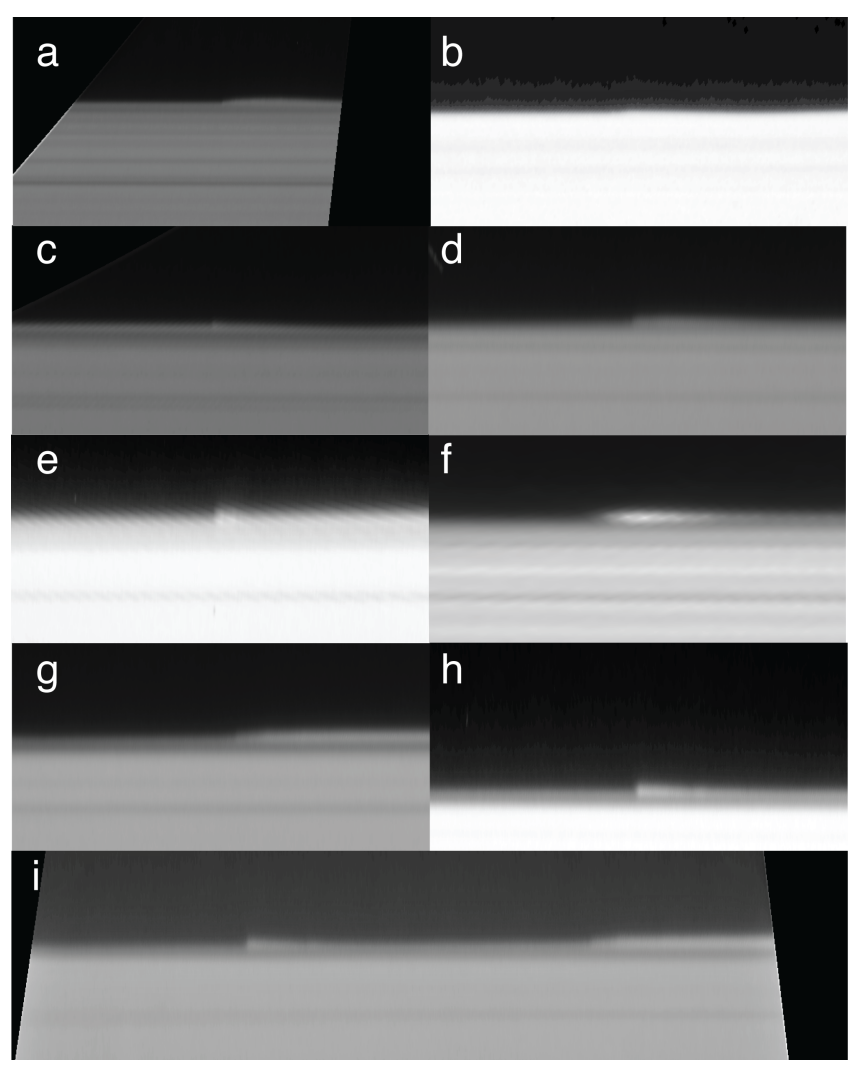

Figure 2: Nine representative reprojected images of the edge of the A ring showing evidence for an embedded object. Each image covers $2^{\circ}$ of longitude approximately centered on the object and a radial range of $300 \mathrm{~km}$ from $136,600 \mathrm{~km}$ to $136,900 \mathrm{~km}$, except for (i) which covers $4^{\circ}$ of longitude. (a) N1717523826 taken on 2012 June 4; (b) N1723600320 taken on 2012 August 14; (c) N1727069080 taken on 2012 September 23; (d) N1729028339 taken on 2012 October 15; (e) N1736819769 taken on 2013 January 14; (f) N1744738746, the discovery image, taken on 2013 April 15; (g) N1750407088 taken on 2013 June 20 showing Object 1; (h) N1750407470 taken on 2013 June 20 showing Object 2; (i) N1750560912 taken on 2013 June 22 showing Objects 1 (right) and 2 (left) separated by $1.60^{\circ}$. Several of the reprojected images have been enhanced to bring out the azimuthal structure. 
data set to search for similar objects in order to estimate the number of such objects at any given time. In each image the region near the edge of the A ring between 136,600 $\mathrm{km}$ and 136,900 km was reprojected using a uniform scale of 1 pixel per $\mathrm{km}$ in radius and 300 pixels per degree of longitude. Such a reprojection produces an aspect ratio of $\sim 8$ that makes radial distortions easier to detect. Each reprojected image was inspected for any localised, radial distortion $\gtrsim 10 \mathrm{~km}$. In cases where multiple images of a fixed range of inertial longitude were obtained, we searched for any localised feature at the level of one pixel that could be tracked from image to image. A total of 107 such detections were made in images obtained between 2012 May 23 and 2013 November 23. Immediately prior to the earlier epoch the spacecraft was in a near-equatorial orbit for 18 months making ring observations difficult. A representative selection of reprojected images of the detected features is shown in Fig. 2. In most cases a feature was detectable as either a discontinuity or a localised peak or trough at the ring edge (see examples in Fig. 2). In each case a measurement of the relevant pixel was converted to an inertial longitude using the known geometry.

\section{Orbital evolution}

We believe that the features we have detected indicate the presence of unresolved, embedded objects near the edge of the A ring. As in the case of 'propellers', tracking the features enables us to determine the semi-major axes of the objects that produce them. Figure 3 shows a plot of the longitude residuals (observed minus calculated) of all the recently detected objects as a function of time relative to a fixed orbit with a semimajor axis of $136,775.20 \pm 0.03 \mathrm{~km}$ (cf. the current [2013 early January] semimajor axis of the edge of the A ring at $136,783 \mathrm{~km}$ ). Although open to interpretation, we believe that the plot reveals patterns that are consistent with the orbital evolution of several objects. Prior to 2013 January there appears to be a single object with a semimajor axis of $136,773.76 \pm 0.01 \mathrm{~km}$. At or immediately after the epoch of discovery there appears to be two sepa- rate objects, often detectable in the same image, that may have a common origin: Object 1 with a semimajor axis of $136,771.91 \pm 0.18 \mathrm{~km}$ and Object 2 with a semimajor axis of $136,775.20 \pm 0.03 \mathrm{~km}$, the reference value for the purposes of calculating the residuals. We believe that this is strong evidence for the breakup of the precursor object due to a collision or tidal disruption, although we recognize that there can be different interpretations to the patterns shown in Fig. 3. There are additional detections shown in Fig. 3; one set trails Objects 1 and 2 by at least $4^{\circ}$ and are morphologically different from them; another second set has distinctly sharper features and could be related to Object 2 as discussed below.

In addition to the variety of morphologies exhibited by Objects 1 and 2 (extended structure, discontinuities, peaks, troughs, etc.) it should be noted that not every predicted location showed a clear detection. In some cases this was due to a lack of resolution (empirically, images with a radial resolution of $>15 \mathrm{~km} /$ pixel make detection unlikely) but in others it was possible to detect quasi-periodic azimuthal structure at the A ring edge extending over $\sim 5^{\circ}$ in longitude trailing the predicted location of the object. We believe that this is evidence that the observed structure depends on orbital phase implying that the object producing the structure has an eccentricity with respect to the local ring material (see below). We extended our search to look for localised features at the A ring edge in other Cassini images. This produced an additional 62 detections between 2005 May 1 and 2008 November 7. However, we do not believe that it is possible to link any of the earlier detections with Objects 1 and 2 even though the spread in residuals with respect to our reference orbit was always $<90^{\circ}$ implying some degree of clustering. Information on all the detections is available from www . maths. qmul.ac.uk/ carl/aringobject/

\section{Edge dynamics}

A common characteristic of many of the detections in the recent epoch is that the radius of the outer edge of the ring has a discontinuity, corre- 


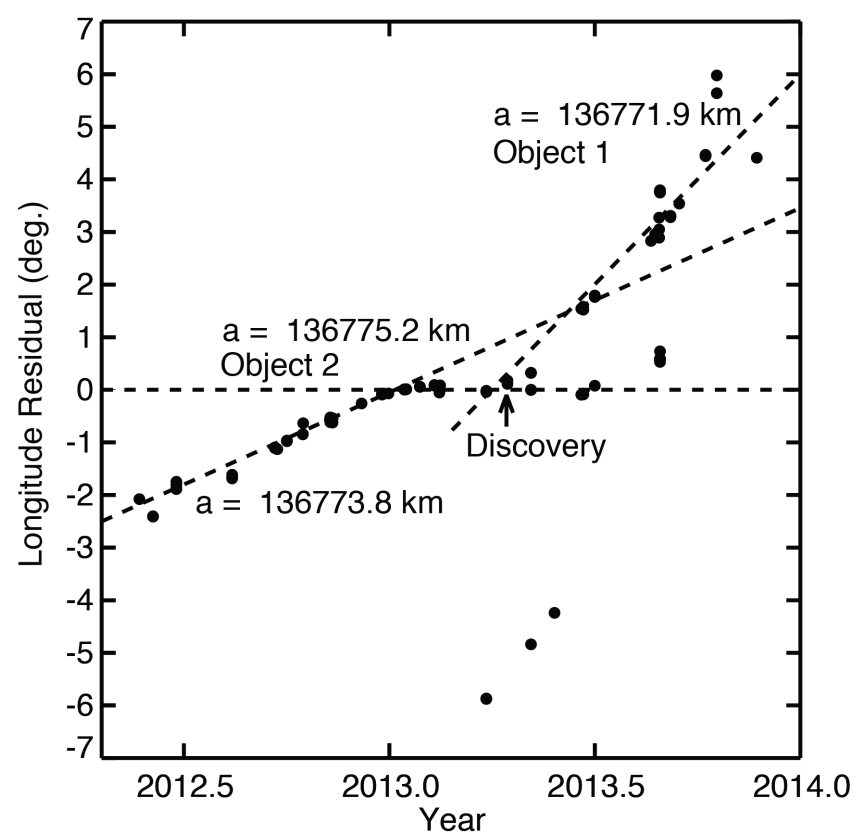

Figure 3: Observed longitudes for object detections since 2012 May 23 with a background reference trend removed. The residuals (observed minus calculated) are with respect to an assumed semimajor axis of $136,775.2 \mathrm{~km}$. This reference trend was derived from a fit to 30 observations, including the discovery image, some pre-discovery images and Object 2. The fitted span is from 2012 December 7 to 2013 June 22. The fitted semimajor axis was $136,775.20 \pm 0.03 \mathrm{~km}$ with a fitted mean motion $604.1833 \pm 0.0002^{\circ} \mathrm{d}^{-1}$ and a mean longitude at the discovery epoch of $198.36 \pm 0.01^{\circ}$. The trend labeled $a=$ $136,773.8 \mathrm{~km}$ comes from a fit to 27 observations between 2012 June 25 and 2013 July 2 including some pre-discovery images and Object 1 (but excluding the discovery image). The trend labeled $a=136,771.9$ comes from a fit to 16 observations between 2013 March 27 and 2013 October 8 (including the discovery image). Other objects distinct from the two primary ones have also been detected and can be distinguished from them by either large residuals or different morphology.
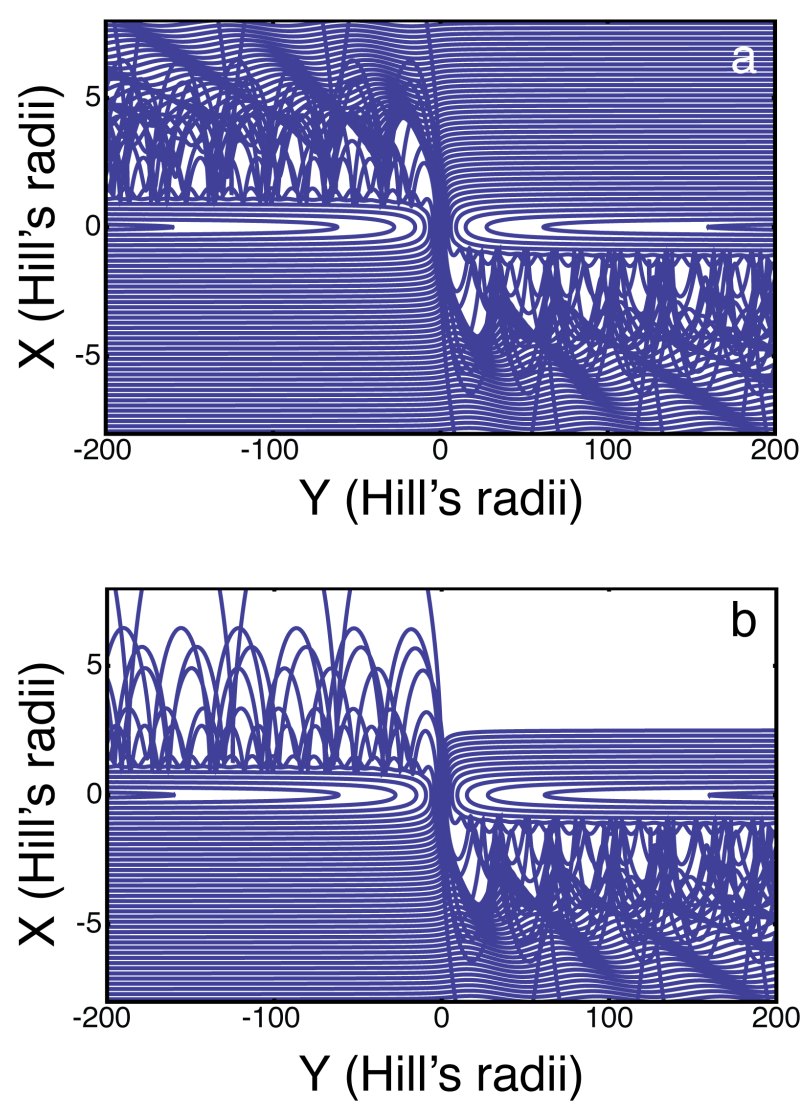

Figure 4: Plots of particle trajectories in the rotating reference frame in the vicinity of an embedded mass in (a) the center of a continuous ring and (b) near the edge of a ring. In this frame ring particles approach the embedded mass from the lower left and upper right. The maximum change in the relative eccentricity occurs for $X=2.5$ and we take this value to define the approximate outer edge of the ring. In this model the discontinuity at $Y=0$ occurs as $X$ jumps from $X \approx 1$ to $X=2.5$. 
sponding to an increase with increasing longitude, as can be seen in Fig. 2. We believe that this can be explained in the context of an embedded object close to the edge of the ring. Figure 4 shows particle trajectories derived by solving Hills equations (see Murray \& Dermott (1999), Chap. 3.13) with an embedded object at the origin. The trajectories produced by an embedded object in a continuous ring (Fig. 4a) give rise to the classical 'propeller' structure. However, those produced by an object near the edge of a ring (Fig. 4b) give rise to a 'half-propeller' structure with a distinct offset due to the absence of particles on external orbits approaching the object. The resulting trajectories shown in the top left of Fig. $4 \mathrm{~b}$ will be too diffuse to be detected thereby producing an obvious discontinuity in longitude at the ring edge, consistent with what is seen in many reprojected images (see Fig. 2d,e,h). In principle the extent of the discontinuity $(\sim 10 \mathrm{~km})$ can be equated to the assumed upper limit $X=2.5 r_{\mathrm{H}}$, where $r_{\mathrm{H}}=\left(m_{\text {object }} / 3 M_{\text {Saturn }}\right)^{1 / 3} a$ is the Hill's radius, to provide an estimate of the size of the embedded object (see Williams (2009)). For an object with the density of Atlas this would imply a mean radius of $3 \mathrm{~km}$ but we consider this to be an overestimate, given the observed gap created by Daphnis (mean radius $4 \mathrm{~km}$ ) perturbing similar ring material. Better estimates of the object's mass and better explanations of the observed structures will have to await detailed dynamical modelling of the interaction between an embedded mass and ring particles in this region.

The outer edge of Saturn's A ring is an unusual location from several points of view. The edge is maintained by the 7:6 inner Lindblad resonance (ILR) with the moon Janus (Porco et al., 1984) and this is observed to create a seven-lobed structure with a radial excursion of $20 \mathrm{~km}$ that rotates with a mean angular velocity (mean motion) close to that of Janus (Porco et al. (1984), Spitale \& Porco (2009)). However, because of Janus' gravitational interaction with its co-orbital moon Epimetheus, the location of the ILR alternates between $a_{\text {outer }}=136,783 \mathrm{~km}$ and $a_{\text {inner }}=$ $136,766 \mathrm{~km}$ every $4.00 \mathrm{y}$ (using data from Jacobson et al. (2008)) although it should be recog- nised that the orbits are constantly affecting one another (Murray \& Dermott (1999), Chap. 3.12). Since January 2010 and throughout the period of time covered in Fig. 3 the resonance has been located at $a_{\text {outer }}$ and therefore we would expect the forced eccentricity on Object 1 due to the 7:6 ILR to be $2 \times 10^{-5}$ (Murray \& Dermott (1999), Eq. 10.21) an order of magnitude smaller than the observed eccentricity of ring particles defining the edge of the A ring (Spitale \& Porco, 2009). Therefore collisions will arise from the relative eccentricity difference between the object and the surrounding ring material.

The effect of the periodic sweeping of the Janus 7:6 ILR over the orbits of the objects discussed in this paper is unpredictable. However, any differences in amplitude and phase between an object's radial excursions and that of the local ring material will result in collisions which could give rise to changes in semimajor axis leading to orbital evolution and this process could easily dominate over changes due to ring or tidal torques at this location. Alternatively, the same mechanism could lead to the destruction of an outwardly evolving object as it continually collides with the ring edge. This may explain the unusual brightness of the object seen in the discovery image and the apparent disappearance of Object 2 soon afterwards. Another possibility is that Object 2 has evolved outwards and no longer exerts a detectable influence on the rings.

\section{Discussion}

The large A ring propeller "Blériot" has been observed to undergo both increases and decreases in semimajor axis over an interval of $4 \mathrm{y}$ (Tiscareno et al., 2010), probably due to stochastic encounters (Crida et al., 2010); indeed, Pan et al. (2012) showed that the Cassini observations are consistent with stochastic migration. However, although it is tempting to see the object reported here as the end state of such an evolutionary process, or even as evidence for the present day operation of the Crida \& Charnoz (2012) mechanism, we believe that it is more likely that it has formed close to where it was discovered. It is known that 
the regular, perturbing action of Prometheus can trigger the formation of aggregations in Saturn's F ring (Beurle et al., 2010). Similarly, the bunching of streamlines produced by the Janus 7:6 ILR (see, e.g. Murray \& Dermott (1999), Fig. 10.9) would create the ideal conditions for the aggregation of ring particles into larger objects. Indeed, high resolution ISS images of the outer A ring taken near the equinox show clear evidence of clumps with vertical extent $<0.5 \mathrm{~km}$ (based on the length of the shadows they cast). Charnoz et al. (2011) showed that Type I migration would lead to the rapid ejection from the rings of any objects denser than that of ice. Therefore, under the reasonable assumption that the formation of clumps sufficiently massive to perturb and evolve due to nearby ring material is an on-going process, we would expect escaped objects to exist in the region exterior to the edge of the A ring. Knowledge of their numbers and fate would help to constrain the rate of production of new objects in Saturn's rings and could confirm the existence of the "pyramidal regime" proposed by Crida \& Charnoz (2012). Continual monitoring of this object and its neighbourhood will provide a unique insight into the processes that govern object formation and orbital evolution in a self-gravitating disk.

\section{Acknowledgments}

This work was supported by the Science and Technology Facilities Council (Grant No. ST / F007566/1) and we are grateful to them for financial assistance. C.D.M. is also grateful to the Leverhulme Trust for the award of a Research Fellowship. The authors thank Jenya Morrice for her help with the image analysis as well as Matthew Hedman and Matthew Tiscareno for useful discussions. We are also grateful to NASA and members of the Cassini project for their help. The original manuscript was considerably improved by helpful comments from Aurélien Crida and Sébastien Charnoz.

\section{References}

Beurle, K., et al., 2010. Direct evidence for gravitational instability and moonlet formation in Saturn's rings.
Charnoz, S., Salmon, J., Crida, A., 2010. The recent formation of Saturn's moon lets from viscous spreading of the win rings. Nature 465, 752-754.

Charnoz, S. et al., 2011. Accretion of Saturn's mid-sized moons during he viscous spreading of young massive rings: Solving the paradox of silicate-poor rings versus silicate-rich moons. Icarus 216, 535-550.

Crida, A., Papaloizou, J.C.B., Rein, H., Charnoz, S., Salmon, J., 2010. Migration of a moonlet in a ring of solid particles: Theory and application to Saturn's propellers. Astron J. 140, 944-953.

Crida, A., Charnoz, S., 2012. Formation of regular satellites from ancient massive rings in the solar system. Science 338, 1196-1199.

Cuzzi, J.N. et al., 2010. An evolving view of Saturn's dynamic rings. Science 327, 1470-1475.

Jacobson, R.A. et al., 2008. Revised orbits of Saturn's small inner satellites. Astron. J. 135, 261-263.

Murray, C.D., Dermott, S.F., 1999. Solar System Dynamics (Cambridge University Press, Cambridge, UK).

Pan, M., Rein, H., Chiang, E., Evans, S.N., 2012. Stochastic flight of propellers. MNRAS 427, 2788-2796.

Porco, C.C., Danielson, G.E., Goldreich, P., Holberg, J.B., Lane, A.L., 1984. Saturn's nonaxisymmteric ring edges at $1.95 R_{\mathrm{S}}$ and $2.27 R_{\mathrm{S}}$. Icarus $60,17-28$.

Porco, C.C. et al., 2004. Cassini imaging science: Instrument characteristics and anticipated scientific investigations at Saturn. Space Sci. Rev., 115, 363-497.

Spitale, J.N., Porco, C.C., 2009. Time variability in the outer edge of Saturn's A-ring revealed by Cassini imaging. Astron. J. 138, 1520-1528.

Thomas, P.C. et al., 2013. The inner small satellites of Saturn: A variety of worlds. Icarus 226, 999-1019.

Tiscareno, M.S. et al., 2006. 100-metre-diameter moonlets in Saturn's A ring from observations of propeller' structures. Nature 440, 648-650.

Tiscareno, M.S., Burns, J.A., Hedman, M.H., Porco, C. C., 2008. The population of propellers in Saturn's A ring. Astron J. 135, 1083-1091.

Tiscareno, M.S. et al., 2010. Physical characteristics and non-Keplerian orbital motion of propeller moons embedded in Saturn's rings. Astrophs. J. 718, L92-L96.

Williams, G.A. 2009, PhD thesis, Queen Mary, Univ. of London. 